\title{
Recomendações multidisciplinares portuguesas sobre o pedido de DXA e indicação de tratamento de prevenção das fraturas de fragilidade
}

Andréa Marques, ${ }^{1}$ Ana M. Rodrigues, ${ }^{2}$ José Carlos Romeu, ${ }^{3}$ Afonso Ruano, ${ }^{4}$ Ana Paula Barbosa, ${ }^{5}$ Eugénia Simões, ${ }^{5}$ Fernanda Águas, ${ }^{6}$ Helena Canhão, ${ }^{7}$ José Delgado Alves, ${ }^{8}$ Raquel Lucas, ${ }^{9}$ Jaime Branco, ${ }^{10}$ Jorge Laíns, ${ }^{11}$ Mário Mascarenhas, ${ }^{12}$ Susete Simões, ${ }^{13}$ Viviana Tavares, ${ }^{14}$ Óscar Lourenço, ${ }^{15}$ J. A. P. da Silva ${ }^{1,16}$

\section{RESUMO}

Objetivo: Estabelecer as recomendações portuguesas relativas à indicação de realização da DXA e de iniciação de tratamento para a prevenção de fraturas de fragilidade.

Métodos: Foi reunido um painel multidisciplinar, representando o leque das especialidades médicas e associações de doentes dedicadas à osteoporose, bem como especialistas nacionais neste domínio e em economia da saúde, com o objetivo de desenvolver recomendações com base na evidência científica e no consenso dos especialistas. As decisões foram suportadas por dados epidemiológicos, sócio-económicos e de qualidade de vida obtidos recentemente sobre as fraturas osteoporóticas.

Resultados: Foram desenvolvidas 10 recomendações que abordam as questões de quem deve ser objeto de investigação com a DXA e quem deve ser tratado com terapêutica para prevenção de fraturas. Os limiares para a avaliação e intervenção baseiam-se na análise de custo-eficácia das intervenções em diferentes limiares da probabilidade a dez anos de fratura osteoporótica, calculados com base na versão portuguesa da FRAX ${ }^{\circledR}$ (FRAX ${ }^{\circledR}$ Port) tendo em consideração os dados epidemiológicos e económicos portugueses. Enumeram-se as limitações da $F R A X{ }^{\circledR}$ e, sempre que possível, propõem-se orientações para uma adaptação.

Conclusões: Os limiares de custo-eficácia para realização de DXA e a instituição de terapêutica para a prevenção das fraturas de fragilidade são agora facultados à população portuguesa. Estes limiares são práticos, baseiam-se em dados epidemiológicos e económicos nacionais e em evidência científica e são corroborados por um painel multidisciplinar de especialistas e de instituições científicas. A implementação destas recomendações oferece perspetivas muito promissoras no que diz respeito a uma utilização mais eficaz dos recursos de saúde na prevenção das fraturas osteoporóticas em Portugal.

Palavras-chave: Risco de Fratura; FRAX; Osteoporose; DMO; DXA; Fraturas de Fragilidade; Orientações; Recomendações.

\section{INTRODUÇÃO}

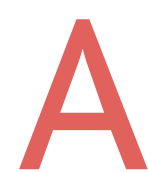

osteoporose (OP) é uma doença metabólica do esqueleto caracterizada por baixa massa óssea e deterioração da microarquitetura que conduz a uma crescente fragilidade óssea e suscetibilidade de fraturas. Em Portugal estima-se que a incidência de fraturas de fragilidade da anca seja de 154 a 572 mulheres em cada 100.000 mulheres e de 77 a 232 em cada 100.000 homens, dependendo da idade. Mais de 10.000 doentes são admitidos todos os anos no Serviço Nacional de Saúde português devido a fraturas de fragilidade da anca, o que acarreta uma despesa total de saúde anual acima de $220 \mathrm{mi}$ - lhões de euros. ${ }^{2}$ Corresponde a $1,4 \%$ do orçamento da saúde para 2013, incluindo serviços privados e públicos, de acordo com as estatísticas de saúde portuguesa. ${ }^{3}$ A despesa total com as fraturas de fragilidade será muito superior, dado que, de acordo com um estudo recente, as fraturas da anca representam apenas cerca de $39,1 \%$ do número total de fraturas de fragilidade observadas em Portugal. ${ }^{4}$

No seu conjunto, as fraturas osteoporóticas representam atualmente um enorme encargo social e económico em Portugal, apesar de o país ter uma das mais baixas incidências da Europa Ocidental. ${ }^{1}$ A dimensão deste problema tende a aumentar cada vez mais, devido ao enve- 
lhecimento progressivo da população e a outras mudanças da sociedade, ${ }^{5}$ a menos que sejam adotadas medidas preventivas eficazes.

O presente artigo sumaria o trabalho do Comité de Especialistas que se reuniu com o objetivo de estabelecer medidas preventivas, facultando aos profissionais de saúde recomendações práticas e válidas relativas ao início de tratamento farmacológico para a osteoporose e/ou para a realização de densitometria (DXA), de forma a otimizar a eficiência das intervenções e a minimizar os custos e riscos para as pessoas e a sociedade.

Desde $2007,{ }^{6}$ data da última publicação das recomendações para o diagnóstico e o tratamento da osteoporose em Portugal, a ferramenta FRAX® foi desenvolvida e incorporada nas orientações clínicas para a OP em vários países. ${ }^{5,7-}$ ${ }^{12} \mathrm{Na}$ verdade, mais de metade das pessoas que sofreram uma fratura de fragilidade não tem OP tal como definida pela densidade mineral óssea (DMO). ${ }^{13}$ A ferramenta FRAX® integra um conjunto de fatores de risco de fratura bem estabelecidos, independentemente da DMO: idade, sexo, índice de massa corporal, fraturas de fragilidade anteriores, antecedentes familiares de fratura, utilização prolongada de glucocorticoides, artrite reumatoide, causas secundárias de osteoporose, tabagismo e consumo de álcool atual, com ou sem DMO. A ferramenta faculta uma estimativa do risco de fratura major osteoporótica (fratura da anca, coluna vertebral, úmero ou antebraço) e de fratura da anca nos dez anos subsequentes. ${ }^{14-15}$ A ferramenta FRAX® fornece esti-

'Serviço de Reumatologia, Centro Hospitalar e Universitário de Coimbra, Coimbra Portugal.

${ }^{2}$ Em representação da Sociedade Portuguesa de Reumatologia (SPR).

${ }^{3}$ Serviço de Reumatologia, Hospital de Santa Maria, Lisboa, Portugal.

${ }^{4}$ Em representação da Sociedade Portuguesa de Ortopedia e de Traumatologia (SPOT).

${ }^{5}$ Em representação da Sociedade Portuguesa de Osteoporose e Doenças Ósseas Metabólicas (SPODOM)

${ }^{6}$ Em representação da Sociedade Portuguesa de Ginecologia (SPG).

${ }^{7}$ Unidade de Investigação em Reumatologia, Instituto Medicina Molecular, Universidade de Lisboa, Hospital de Santa Maria, Lisboa, Portugal.

${ }^{8}$ Serviço Medicina 4, Hospital Prof. Doutor Fernando Fonseca. CEDOC/NOVA Escola Médica. Em representação da Sociedade Portuguesa de Medicina Interna (SPMI). ${ }^{9}$ EPIUnit, Instituto de Saúde Pública, Universidade do Porto. Em representação do Observatório Nacional das Doenças Reumáticas (ONDOR).

${ }^{10} \mathrm{Centro}$ Hospitalar de Lisboa Ocidental. CEDOC/NOVA Escola Médica.

${ }^{11} E m$ representação da Sociedade Portuguesa de Medicina Física e de Reabilitação (SPMFR).

${ }^{12} E m$ representação da Sociedade Portuguesa de Endocrinologia, Diabetes e Metabolismo (SPEDM).

${ }^{13} \mathrm{Em}$ representação da Associação Portuguesa de Medicina Geral e Familiar (APMGF)

${ }^{14} \mathrm{Em}$ representação da Associação Nacional contra a Osteoporose (APOROS).

${ }^{15}$ Centro de Estudos e Investigação em Saúde, Universidade de Coimbra (CEISUC).

Faculdade de Economia, Universidade de Coimbra, Coimbra, Portugal.

${ }^{16}$ Clínica Universitária de Reumatologia, Faculdade de Medicina, Universidade de Coimbra, Coimbra, Portugal. Investigador Principal. mativas válidas sem valores DMO, ${ }^{16-17}$ embora a sua eficácia aumente quando a DMO também é considerada. ${ }^{18}$ Este algoritmo incorpora a epidemiologia de fraturas e a taxa de mortalidade de cada país para facultar estimativas de probabilidade de fratura ajustadas à população onde está a ser aplicado. A ferramenta FRAX® foi construída tendo por base vários estudos de coorte na Europa, América do Norte, Ásia e Austrália, foi validada em 62 países e adotada por muitos como base fundamental das decisões sobre quem tratar.

Com isto em mente foi recentemente validado o modelo FRAX para a avaliação da probabilidade de fratura osteoporótica na população portuguesa / - FRAX®Port ${ }^{15}$ (http://www.shef.ac.uk/FRAX/tool.aspx?country=53).

Através de uma revisão sistemática da literatura com meta-análise ${ }^{19} \mathrm{e}$ do consenso obtido após discussão, chegamos à conclusão de que o FRAX® é a ferramenta mais apropriada para alcançar os objetivos similares em Portugal. Entre as suas vantagens destaca-se a possibilidade de ser utilizado mesmo no caso de ausência da DMO, permitindo o seu resultado decidir quando a DXA é necessária.

Foi ainda realizada uma avaliação cuidadosa, a nível de todo o país, dos custos das fraturas de anca e do seu impacto na qualidade de vida e na mortalidade. ${ }^{2}$ As probabilidades de risco de fratura acima das quais as diferentes intervenções se tornam eficazes em termos de custos, no contexto português atual, foram definidas com base em metodologia económica coesa, apoiada por especialistas internacionalmente reconhecidos. ${ }^{2}$

A realização deste trabalho permitiu uma base sólida para se efetuar uma revisão oportuna das recomendações portuguesas relativas ao limiar de risco para realização da DXA e início de tratamento farmacológico da osteoporose.

Com base no que foi exposto, recomenda-se que as decisões relativas à eficácia da DXA ou do início do tratamento sejam tomadas com base em estimativas de risco real de fratura, tendo por base as implicações económicas das fraturas e as diferentes estratégias preventivas.

O presente relatório não cobre todas as opções de tratamento possíveis e não pretende substituir a responsabilidade individual do médico para com o doente ou a escolha pessoal de cada doente. Os autores desejam sublinhar que não é possível apresentar uma orientação formal para cada situação específica ou co-morbilidade por falta de evidência apropriada. O juízo clínico é necessário nestas condições.

Este trabalho, bem como a série de estudos de apoio já publicados ou em vias de publicação, foi financiado pelo Governo português através da Direção-Geral da Saúde (DGS), de acordo com uma proposta apresentada pela As- 
sociação Nacional Contra a Osteoporose (APOROS) e através de um subsídio da Amgen. Nenhum dos financiadores teve qualquer envolvimento na conceção dos estudos, na interpretação dos resultados ou no conteúdo dos relatórios e recomendações que deles resultaram.

Foi elaborado um total de dez recomendações (Quadro I).

\section{MÉTODOS}

\section{Desenvolvimento das orientações}

Vários especialistas nacionais em osteoporose e todas as sociedades científicas portuguesas com interesse na osteoporose foram convidadas e aceitaram participar no desenvolvimento das presentes recomendações: Reumatologia, Ortopedia e Traumatologia; Endocrinologia, Diabetes e Metabolismo; Ginecologia; Medicina Interna; Medicina Física e de Reabilitação; Medicina Familiar; Observatório Nacional das Doenças Reumáticas e a Sociedade Portuguesa de Osteoporose e Doenças Ósseas Metabólicas. A única organização nacional de doentes ativa no domínio da osteoporose, a Associação Nacional contra a Osteoporose (APOROS), também participou no Comité. O Comité era constituído por dezassete membros, todos com direito a voto, tendo todos sido coautores do presente relatório.

As questões relevantes a serem abordadas pelas recomendações foram definidas por consenso numa primeira ronda de consulta por correio eletrónico, de acordo com uma proposta preparada pelo investigador principal (JAPS) e pelo bolseiro de investigação (AM). Para o tratamento de cada questão foi realizada uma revisão aprofundada da literatura (AM e JAPS), que foi disponibilizada aos membros do comité antes da reunião. A pesquisa eletrónica foi realizada na PubMed/MEDLINE (2006 - 15 de janeiro de 2015). As estratégias de pesquisa incluíram as seguintes descritores: 'Osteoporosis', 'Osteoporotic fractures,' 'RiskAssessment,' 'Algorithms,' 'Recommendations, 'Guidelines,' 'Treatment', 'Cost-effectiveness,' 'Bone Mineral Density' and 'DXA'. Foram incluídos nesta revisão artigos originais, revisões e orientações relativas aos limiares para o início do tratamento e pedido de DXA. As referências citadas nas revisões sistemáticas publicadas ou os artigos originais também foram analisadas.

Tendo por base os dados recolhidos, o investigador principal preparou possíveis respostas às questões selecionadas e submeteu-as, juntamente com esses dados, ao Comité de Especialistas numa segunda ronda de $\boldsymbol{e}$-mails. Solicitou-se aos membros do Comité que apreciassem os dados enviados e as recomendações ou que propusessem novas recomendações. Numa terceira ronda de $e$-mails, antes da reu- nião final presencial, todas as alternativas propostas foram submetidas à consideração dos membros do Comité.

A reunião para discussão dos dados obtidos, votar as respostas possíveis e, assim, criar um conjunto de recomendações teve lugar no dia 13 de março de 2015. A reunião foi devidamente registada, para documentação e clarificação futura de dúvidas. Os votos dos representantes individuais e o grau de acordo relativamente a cada recomendação foram também registados. Os dados portugueses relativos à relação custo-eficácia das intervenções, segundo os diferentes limiares de risco, foram revelados ao painel pela primeira vez, só depois de terem sido estabelecidos definitivamente todos os princípios orientadores. Apenas os três membros que conduziram o estudo (AM, OL, JAPS) tinham conhecimento destes dados. Esta estratégia foi adotada para garantir que a base de custo-eficácia para a decisão de intervir estava fundamentada nos princípios orientadores e não tinha sido influenciada por considerações sobre a percentagem de população elegível para a intervenção, os seus custos globais ou a (não) similaridade dos limiares de intervenção portugueses relativamente a outras orientações publicadas.

Houve uma ronda final de $\boldsymbol{e}$-mails para aperfeiçoamento de algumas recomendações.

Por último, este documento foi preparado e circulou entre os membros do comité até se alcançar a sua versão final, que foi submetida à aprovação e aval das referidas sociedades e associações.

\section{Definições concetuais subjacentes: princípios orientadores}

Como fase preparatória para a definição das recomendações, o Comité preparou e desenvolveu uma discussão pormenorizada com vista a estabelecer princípios e conceitos de orientação que se apresentam de seguida:

\section{- Princípio orientador 1.}

Os fatores de risco para a osteoporose, como os relativos à dieta, exercício, exposição ao sol, terapêutica farmacológica, devem ser avaliados pelos profissionais de saúde e pelos doentes durante toda o ciclo vital e devem ser corrigidos, quando apropriado.

[Este princípio orientador foi aprovado por todos os membros do comité com 17 votos em 17.]

Muitos fatores de risco para a osteoporose influenciam a saúde dos ossos desde muito cedo e durante toda a vida, mesmo se as consequências da osteoporose só se tornarem aparentes mais tarde na vida. É o caso, por exemplo, da 


\begin{tabular}{|c|c|c|}
\hline Recomendação & Votos & $\begin{array}{l}\text { Média do } \\
\text { acordo }\end{array}$ \\
\hline $\begin{array}{l}\text { 1. A implementação de medidas gerais, não farmacológicas, de prevenção da osteoporose, } \\
\text { como dieta, suplementação com vitamina } D \text {, exercício, prevenção de quedas e } \\
\text { monitorização da utilização de terapêutica para os ossos, deve ser aplicada a todas as } \\
\text { idades sempre que sejam identificados fatores de risco corrigíveis, independentemente da } \\
\text { FRAX }{ }^{\circledR} \text { e DMO. }\end{array}$ & $\begin{array}{r}\text { Aprovado por } 17 \\
\text { votos em } 17\end{array}$ & $97 \%(75-100)$ \\
\hline $\begin{array}{l}\text { 2. O tratamento farmacológico da osteoporose deve ser recomendado, exceto se } \\
\text { contraindicado, a todas as pessoas com idade superior a } 50 \text { anos que já sofreram de: } \\
\text { A. } \geq 1 \text { Fratura de fragilidade da anca ou } \geq 1 \text { fratura sintomática de fragilidade vertebral; } \\
\text { ou } \\
\text { B. } \geq 2 \text { Fraturas de fragilidade, independentemente da localização da fratura ou da } \\
\text { ausência de sintomas (e.g., duas fraturas assintomáticas vertebrais). }\end{array}$ & $\begin{array}{r}\text { Aprovado por } 17 \\
\text { votos em } 17\end{array}$ & $95,6 \%(70-100)$ \\
\hline $\begin{array}{l}\text { 3. Todos homens e mulheres portugueses com mais de } 50 \text { anos de idade devem } \\
\text { submeter-se a uma avaliação do risco a dez anos de fratura osteoporótica através da } \\
\text { ferramenta FRAX }{ }^{\circledR} \text { Port, com ou sem DXA. }\end{array}$ & $\begin{array}{r}\text { Aprovado por } 17 \\
\text { votos em } 17\end{array}$ & $95,9 \%(80-100)$ \\
\hline $\begin{array}{l}\text { 4. Para estimativas do FRAX }{ }^{\circledR} \text { Port, sem DXA, entre } 7 \% \text { e } 11 \% \text { para fraturas osteoporóticas } \\
\text { major E entre } 2,0 \% \text { e } 3,0 \% \text { para fraturas da anca o índice } T \text { do cabeça do fémur avaliado } \\
\text { e inserido no FRAX }{ }^{\circledR} \text { Port (Figura } 2 \text { ). A DXA pode ser justificada em certas condições }\end{array}$ & $\begin{array}{l}\text { Aprovado por } 16 \\
\text { votos favoráveis } \\
\text { e } 1 \text { abstenção }\end{array}$ & $90,9 \%(60-100)$ \\
\hline $\begin{array}{l}\text { 5.A. Nos homens e mulheres com uma estimativa de risco de fratura (sem DMO) inferior } \\
\text { a } 7 \% \text { para as fraturas osteoporóticas major E } 2 \% \text { para a fratura da anca, a decisão de não } \\
\text { tratar com agentes farmacológicos pode ser justificada, sem necessidade de realizar uma } \\
\text { DXA. Medidas gerais de prevenção devem ser postas em prática. }\end{array}$ & $\begin{array}{l}\text { Aprovado por } 16 \\
\text { votos favoráveis } \\
\text { e } 1 \text { abstenção }\end{array}$ & $95 \%(50-100)$ \\
\hline $\begin{array}{l}\text { 5.B. Nestes casos, a avaliação FRAX }{ }^{\circledR P} \text { Port deve ser repetida com uma frequência que } \\
\text { depende da proximidade da avaliação anterior do limite inferior de indicação para a DXA } \\
\text { e igualmente da ocorrência de mudanças significativas nos fatores de risco clínicos } \\
\text { (Figura } 2 \mathrm{~A} \text { ). }\end{array}$ & $\begin{array}{l}\text { Aprovado por } 16 \\
\text { votos favoráveis } \\
\text { e } 1 \text { abstenção }\end{array}$ & $93,8 \%(60-100)$ \\
\hline $\begin{array}{l}\text { 6. Nos homens e mulheres com uma estimativa de risco de fratura, sem DXA, superior a } \\
11 \% \text { para as fraturas osteoporóticas major ou de } 3 \% \text { para as fraturas da anca, o tratamento } \\
\text { farmacológico com o alendronato genérico é custo-eficaz e deve ser aconselhado (salvo } \\
\text { em caso de contraindicação), sem necessidade de realizar DXA (Figura 2A). }\end{array}$ & $\begin{array}{l}\text { Aprovado por } 16 \\
\text { votos favoráveis } \\
\text { e } 1 \text { abstenção }\end{array}$ & $95,3 \%(80-100)$ \\
\hline $\begin{array}{l}\text { 7. Nos homens e mulheres com uma estimativa FRAX }{ }^{\circledR} \text { Port de risco a dez anos, com DXA, } \\
\text { igual ou superior a } 9 \% \text { para as fraturas osteoporóticas major ou a } 2,5 \% \text { para as fraturas } \\
\text { da anca, o tratamento farmacológico para a osteoporose com o alendronato genérico é } \\
\text { custo-eficaz e deve ser aconselhado (salvo em caso de contraindicação) (Quadro I e } \\
\text { Figura 2B). }\end{array}$ & $\begin{array}{r}\text { Aprovado por } 17 \\
\text { votos em } 17\end{array}$ & $93,2 \%(60-100)$ \\
\hline $\begin{array}{l}\text { 8. A decisão de iniciar um tratamento antiosteoporótico com outros medicamentos, que } \\
\text { não o alendronato genérico, deve ser justificada pelos respetivos limiares de intervenção } \\
\text { de custo-eficácia (Quadro IV). }\end{array}$ & $\begin{array}{l}\text { Aprovado por } 16 \\
\text { votos favoráveis } \\
\text { e } 1 \text { abstenção }\end{array}$ & $88,1 \%(0-100)$ \\
\hline
\end{tabular}




\begin{tabular}{|c|c|c|}
\hline Recomendação & Votos & $\begin{array}{l}\text { Média do } \\
\text { acordo }\end{array}$ \\
\hline $\begin{array}{l}\text { 9. A. Nos homens e mulheres com uma estimativa FRAX®Port de risco a dez anos, com } \\
\text { DXA, inferior a } 9 \% \text { para as fraturas osteoporóticas major E inferior a } 2,5 \% \text { para as } \\
\text { fraturas da anca, os agentes farmacológicos não são custo-eficazes e a decisão de não os } \\
\text { utilizar pode estar justificada. As medidas gerais de prevenção aplicáveis devem ser postas } \\
\text { em prática. }\end{array}$ & $\begin{array}{r}\text { Aprovado por } 17 \\
\text { votos em } 17\end{array}$ & $96,5 \%(80-100)$ \\
\hline $\begin{array}{l}\text { 9.B. Nestes doentes, as avaliações DXA e FRAX }{ }^{\circledR} \text { Port devem ser repetidas a cada dois anos } \\
\text { ou sempre que os fatores de risco clínicos sofram uma alteração significativa (Figura 2). A } \\
\text { DXA pode não ser necessária se os valores de uma DMO anteriores forem tranquilizadores. }\end{array}$ & $\begin{array}{l}\text { Aprovado por } 16 \\
\text { votos favoráveis } \\
\text { e } 1 \text { abstenção }\end{array}$ & $92,8 \%(75-100)$ \\
\hline $\begin{array}{l}\text { 10. Quando recorrerem ao FRAX }{ }^{\circledR} \text { Port para a implementação destas recomendações, os } \\
\text { profissionais de saúde devem estar conscientes das limitações deste instrumento e } \\
\text { ponderarem a necessidade de ajustar as estimativas de risco fornecidas por esta } \\
\text { ferramenta, em situações específicas que se descrevem no texto. }\end{array}$ & $\begin{array}{r}\text { Aprovado por } 17 \\
\text { votos em } 17\end{array}$ & $97,6 \%(70-100)$ \\
\hline
\end{tabular}

dieta (cálcio, proteínas), exercício, níveis da vitamina D e terapêutica, como os glucocorticoides. Todas estas condições têm implicações sobre a saúde que vão muito para além dos limites da saúde do osso e devem, portanto, ser consideradas como uma rotina médica. A correção destes fatores de risco é uma parte integrante da gestão da osteoporose, sendo geralmente referida como «Medidas Gerais».

\section{- Princípio orientador 2.}

A decisão de prescrever um tratamento farmacológico para a osteoporose deve basear-se no risco a dez anos de um doente desenvolver uma fratura osteoporótica estimado pela ferramenta FRAX ${ }^{\circledR}$ Port.

[Este princípio orientador foi aprovado por todos os membros do comité (17/17).]

O FRAX® é um algoritmo desenvolvido sob os auspícios da Organização Mundial de Saúde e permite estimar o risco individual de fraturas osteoporóticas durante os dez anos subsequentes, com base em onze fatores de risco clínicos (FRC), tendo a sua influência sido demonstrada, independentemente da DMO, através de estudos individuais e de meta-análises. Estes fatores de risco estão facilmente disponíveis na prática clínica: idade, peso, altura, fragilidade anterior às fraturas, antecedentes familiares de fratura da anca, tabagismo atual, $\geq 3$ meses de utilização de glucocorticoides, artrite reumatoide, causas de osteoporose secundária (diabetes tipo I, osteogenesis imperfecta em adultos, hipertiroidismo de longa data não tratado, hi- pogonadismo e menopausa prematura $(<45$ anos), subnutrição crónica ou absorção deficiente e doença crónica do fígado) e consumo de álcool. A ferramenta FRAX® pode ser utilizada com ou sem DMO (Figura 1).

Quando o cálculo utiliza apenas FRC, isto é, não considera a DMO, a ferramenta FRAX® revelou ter um melhor desempenho do que a DMO sozinha na predição de fratura major. ${ }^{20} \mathrm{O}$ desenvolvimento desta ferramenta teve por base uma excelente metodologia ${ }^{14} \mathrm{e}$ a sua validade foi confirmada externamente, até agora por vinte e seis estudos realizados em diferentes países e coortes. ${ }^{14,21-43}$ Um total de 62 países e/ou etnias tem o modelo disponível e muitos outros estão a ser desenvolvidos. ${ }^{5}$

Uma revisão sistemática da literatura e meta-análise realizada recentemente por alguns membros do Comitét ${ }^{44}$ demonstra claramente que o FRAX é a ferramenta mais robusta e acessível para prever o risco de fraturas osteoporóticas. A sua precisão está muito bem estabelecida e demonstrada por AUCs da análise ROC para as previsões de fratura, que se situam entre 0,71 a 0,79 na meta-análise. Este desempenho só foi ultrapassado pela ferramenta QFracture, ${ }^{44}$ mas esta ferramenta exige a consideração de 31 fatores de risco clínicos e apenas foi validada para o Reino Unido e Irlanda.

A ferramenta FRAX®Port é a versão portuguesa do FRAX®, desenvolvido para incorporar a atual epidemiologia das fraturas da anca e a mortalidade da população geral portuguesa. ${ }^{15}$ A metodologia e os resultados desta adaptação foram validados pela Organização Mundial de Saúde atra- 


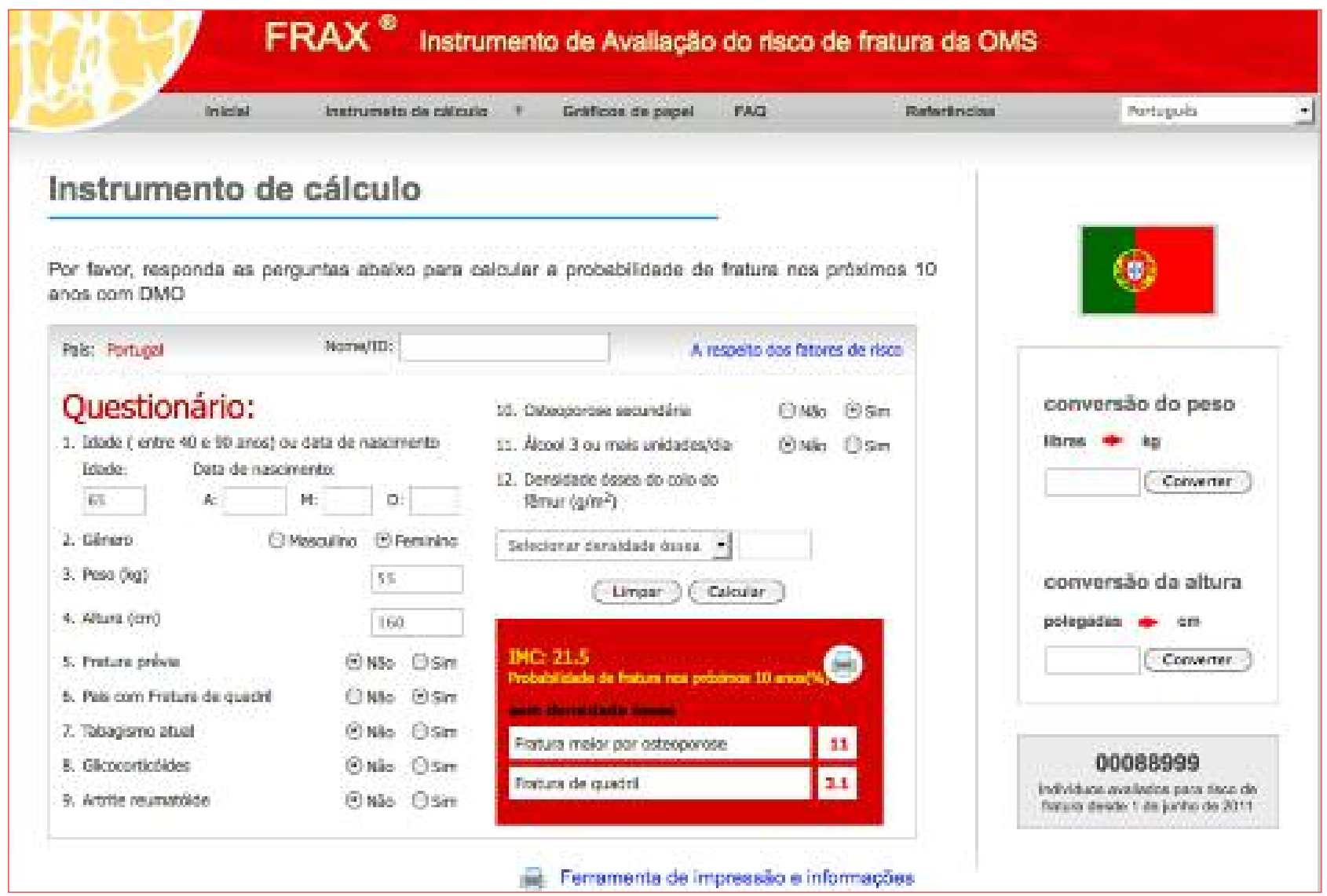

Figura 1. Página do ecrã para a entrada dos dados e a avaliação de risco de fratura na versão portuguesa da ferramenta FRAX® ${ }^{\circledR}$ (Modelo português, versão 3.9., http://www.shef.ac.uk/FRAX/tool.aspx? country=53).

[Com a autorização da Organização Mundial de Saúde em colaboração com o Centre for Metabolic Bone Diseases, Faculdade de Medicina, Universidade de Sheffield.]

vés do centro de cooperação responsável pela criação do FRAX® e por todas as sociedades científicas e organizações de doentes com interesse em osteoporose em Portugal. A ferramenta FRAX®Port está facilmente disponível online.

\section{- Princípio orientador 3.}

A presença de fraturas de fragilidade prévias justifica a consideração de tratamento farmacológico, independentemente da estimativa de risco do FRAX®Port.

[Este princípio orientador foi aprovado com 15 votos favoráveis, 1 voto contra e 1 abstenção.]

Vários estudos corroboram a conclusão de que é economicamente eficaz tratar pessoas com uma fratura de fragilidade prévia da anca ou vertebral..$^{8-9,45}$ As fraturas das vertebrais, por exemplo, constituem um fator de risco muito importante para uma fratura subsequente vertebral ou da anca, ${ }^{46-47}$ enquanto as fraturas do antebraço predizem uma futura fratura da vertebral ou da anca. ${ }^{48}$

$O$ voto contra justificou-se com base no facto de as fraturas anteriores já serem consideradas no FRAX®.

O intervalo de tempo desde a última fratura prévia também é relevante: o risco de fraturas futuras é maior durante os primeiros 2-3 anos, mas continua a ser significativamente elevado por um período de 10 a 15 anos (muito especialmente para as fraturas do fémur proximal, as fraturas de vertebrais e as fraturas do úmero). ${ }^{49-50}$

\section{- Princípio orientador 4.}

Os médicos devem estar conscientes das limitações do FRAX® e da DXA e devem introduzir adaptações judiciosas e informadas na avaliação do risco de fratura, quando essas limitações estão presentes.

[Este princípio orientador foi aprovado por todos os membros do comité - 17 votos em 17.] 


\section{- Princípio orientador 5.}

Os limiares de intervenção portuguesa devem basear-se numa avaliação do FRAX® a dez anos semelhante para todas as idades. Este princípio só deve ser posto de lado se as avaliações sócio-economicas demonstrarem que o limiar de intervenção para um determinado grupo de idade e sexo difere em mais de 50\% do valor recomendado com base na população total.

[Este princípio orientador foi aprovado por 10 dos 17 membros do comité, 4 votos contra e 3 abstenções.]

Este ponto foi um dos mais controversos na reunião de consenso. A recomendação final é semelhante às orientações adotadas pela Fundação Nacional para a Osteoporose dos EUA $^{12}$ e do Canadá. ${ }^{11}$ Para estes dois países, o limiar de intervenção foi definido como o nível de risco acima do qual o custo por QALY ganho se encontrava dentro de limites nacionais aceitáveis. Para as duas orientações foi adotado um valor similar de risco estimado de fratura como limiar de intervenção para todas as idades e para ambos os sexos, apesar de haver pequenas diferenças em função da idade e do sexo em todos os níveis de risco que definem a relação de custo-eficácia.

As recomendações emitidas pelo Royal College of Physicians do Reino Unido, ${ }^{45}$ pela Associação contra a Osteoporose da Suíça ${ }^{9}$ e pela Autoridade Nacional de Saúde de França $^{7}$ seguiram uma abordagem concetual diferente: o tratamento é recomendado para todas as pessoas cujo risco a dez anos, estimado pelo FRAX®, seja igual ou superior ao de um doente da mesma idade que já sofreu uma fratura de fragilidade. Este conceito baseia-se no facto de o tratamento de pessoas com uma fratura de fragilidade ter revelado ser economicamente eficaz. Uma vez que o risco de fratura aumenta com a idade, todos os outros fatores, sendo iguais, a abordagem determina que o limiar de intervenção aumente substancialmente com a idade. Por exemplo, de acordo com as orientações do Reino Unido acima referidas, o tratamento será recomendado para pessoas de 50 anos de idade cujo risco de fratura a dez anos seja de 7,5\%, mas não para uma pessoa de 70 anos de idade cujo risco estimado a dez anos é de $24 \%$. A maioria do nosso comité recusou esta abordagem filosófica. Baseou-se, sobretudo, no argumento de que um QALY ganho deve ter o mesmo valor para todas as idades. Sublinha-se ainda que a idade e a mortalidade já são consideradas no FRAX®e, por essa razão, influenciam a avaliação do risco de fratura. De uma forma geral, o comité decidiu, por maioria, manter o conceito segundo o qual, por razões de equidade, poupanças similares na saúde, medidas pelos QALY, devem justificar esforços financeiros similares por parte da sociedade, independentemente da idade.
- Princípio orientador 6.

Os limiares de intervenção nacionais devem basear-se em dados de relação custo-eficácia.

[Este princípio foi aprovado por todos os membros do comité - 17 votos em 17.]

Ao proceder assim, o Comité decidiu aceitar que o limiar de intervenção, a nível da população, deve contemplar considerações económicas e não uma perspetiva «política» do nível de risco que poderia justificar uma intervenção, independentemente dos seus custos e da vontade da sociedade de suportar estes custos. Desta forma, o Comité leva em consideração que o preço da intervenção e a disponibilidade da sociedade para pagar precisam de ser tomados em linha de conta nas decisões de tratar ou de não tratar.

Este princípio implica que as decisões de tratar devem ter o mesmo fundamento em todos os domínios da medicina no nosso país - o impacto das intervenções em termos de QALY ganhos deve ser calculado, o custo por QALY poupado (ou Rácio Custo-Efetividade Incremental-RCEI) deve ser determinado e, naturalmente, uma mesma disponibilidade para pagar por um QALY deve ser aplicada, qualquer que seja a doença e a intervenção em causa.

\section{- Princípio orientador 7.}

Os limiares de intervenção devem ser baseados em dados que reflitam a realidade portuguesa no que respeita a fraturas, mortalidade, custos e eficácia de tratamento.

[Este princípio orientador foi aprovado por todos os membros do comité - 17 votos em 17.]

As recomendações sobre o nível de risco de fratura acima das quais a intervenção farmacológica se torna custoeficaz estão dependentes de dimensões que variam muito consideravelmente a nível nacional, como: epidemiologia das fraturas, mortalidade geral, mortalidade associada às fraturas, intervenções médicas em caso de fraturas, custos de tratamento das fraturas, custos das intervenções de prevenção, políticas de saúde, custos por QALY ganho (ICER), situação económica do país e disponibilidade para pagar. É necessário considerar os dados nacionais quando se tomam tais decisões e requer que as recomendações sobre o limiar de intervenção para Portugal tenham que aguardar até que tais dados estejam disponíveis.

\section{- Princípio orientador 8.}

O limiar de tratamento farmacológico da osteoporose deve ser estabelecido com base em estimativas a dez anos que correspondem a uma disponibilidade de pagar por QALY ganho de $32.000 €$ 
A mais económica de todas as intervenções farmacológicas deve ser tida como base para decidir qual o limiar de intervenção real para a população portuguesa.

[Este princípio orientador foi aprovado por 16 membros do comité e 1 abstenção.]

A relação de custo-eficácia de uma determinada intervenção só pode ser estabelecida através da comparação do seu impacto com um valor fixado para a disponibilidade para pagar por QALY ganho. ${ }^{51}$ Não está estabelecida uma política nacional portuguesa que estabeleça a Disponibilidade para Pagar por QALY. Por esta razão, o painel decidiu adotar as recomendações emitidas pela OMS de que este valor deve ser fixado em duas vezes o Produto Nacional Bruto (PNB) per capita ${ }^{52}-32.000$ €é um número arredondado de 2 x $16.400 €$ o PNB per capita em Portugal para 2014..$^{53}$

A escolha da intervenção mais económica como referência baseia-se no facto de os custos e a eficiência de cada uma das alternativas disponíveis serem considerados aquando do estabelecimento do custo per QALY (RCEI).

Todas as decisões acima indicadas foram tomadas antes de os estudos atuais de custo-eficácia para Portugal terem sido apresentados ao Comité.

\section{- Princípio orientador 9.}

A DXA deve ser realizada quando exista uma probabilidade razoável de ela alterar a decisão de tratar/não tratar que pode ser tomada com base na avaliação de risco do FRAX ${ }^{\circledast}$ Port, feito sem a DXA.

[Este princípio foi aprovado com 16 votos favoráveis e 1 abstenção.]

Acrescentar DXA aos FRC nos resultados do FRAX®, de acordo com a nossa meta-análise, traduz uma melhoria da AUC de 0,74 para $0,79 .{ }^{44} \mathrm{~A}$ DXA também pode ajudar o clínico a aferir a probabilidade de osteoporose secundária, a quantificar a resposta à terapêutica e a motivar o doente para o tratamento. O Comité considera que a realização da DXA, no momento de tomar a decisão de tratar ou não, representa um custo relativamente menor tendo em vista os encargos globais da doença, que são compensados pelas vantagens oferecidas por este exame. Esta perspetiva conduziu a uma recomendação menos rígida sobre quando realizar a DXA:

Com base neste princípio orientador, os seguintes conceitos foram definidos para os objetivos desta recomendação:

- Limiar de intervenção: A estimativa de risco a dez anos do FRAX®Port, com DMO, acima do qual o tratamento farmacológico é justificado.

- Variação do risco de fratura indicando a necessidade de DXA: A variação da estimativa do risco a dez anos do FRAX®Port, sem DMO, dentro da qual DXA é justificada, porque compreende uma probabilidade razoável de mudar a decisão de tratar ou de não tratar.

Idealmente, os limiares inferior e superior para o DXA deveriam basear-se em dados da vida real dos portugueses, estabelecendo a probabilidade de DMO e provocando uma mudança na decisão de tratar/não tratar à volta do limiar de intervenção. Na ausência de tais dados, e tendo em consideração as questões acima descritas, o Comité decidiu, por consenso, estabelecer estes valores em $2 \%$ e $0,5 \%$ acima e abaixo do limiar de intervenção para as fraturas osteoporóticas major e para fraturas da anca, respetivamente.

\section{ANÁLISE DA RELAÇÃO CUSTO-EFICÁCIA}

Uma vez adotados os Princípios de Orientação, foi apresentada ao Comité a análise da relação custo-eficácia do alendronato genérico (a intervenção menos cara) versus nenhum tratamento (Quadro II).

Foi realizado um estudo pormenorizado com uma amostra representativa de doentes portugueses com fraturas da anca para estabelecer o impacto das fraturas osteoporóticas em termos de consumo de recursos (custos diretos e indiretos), mortalidade e qualidade de vida. Foi adotada uma perspetiva societal, isto é, todos os custos foram considerados, independentemente da entidade pagadora ser o doente ou o sistema de segurança social. ${ }^{2}$

Estes dados foram incorporados num modelo económico Markov, previamente validado, ${ }^{54}$ que sintetizou os dados relevantes disponíveis, como a incidência das fraturas e a sua distribuição etária, a mortalidade da população em geral, o custo, eficácia e risco de efeitos adversos das diferentes terapêuticas, a necessidade de co-medicação e procedimentos de seguimento e a adesão terapêutica. Este modelo permite calcular o Rácio Custo-Efetividade Incremental - ICER para cada intervenção, um conceito que pode ser compreendido como o custo pago por cada QALY ganho em comparação com nenhum tratamento. Os resultados foram utilizados para estabelecer os níveis de risco estimado de fratura a partir dos quais uma determinada intervenção se torna economicamente eficiente, i.e., os resultados em custos por QALY dentro de uma disponibilidade para pagar definida.

Com base nos resultados publicados ${ }^{55} \mathrm{o}$ Comité decidiu adotar as estimativas de risco FRAX®Port de 9\% para as fraturas osteoporóticas major e de $2,5 \%$ para as fraturas da anca como limiares de intervenção para o alendro- 


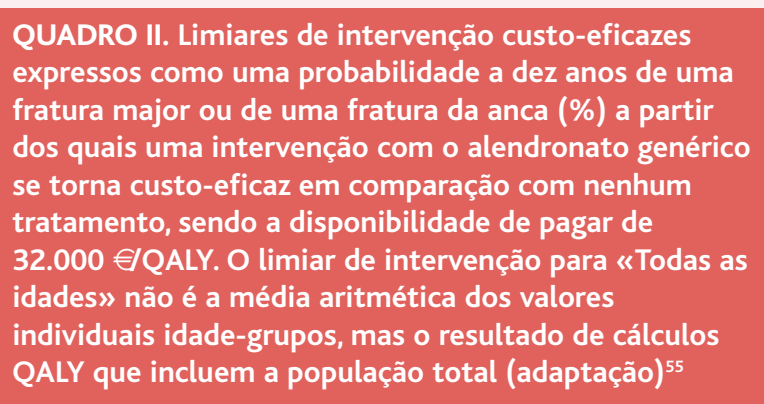

\begin{tabular}{l|c|c} 
Idade & $\begin{array}{c}\text { Probabilidade a 10 } \\
\text { anos de uma fratura } \\
\text { major (\%) }\end{array}$ & $\begin{array}{c}\text { Probabilidade a 10 } \\
\text { anos de uma fratura } \\
\text { da anca (\%) }\end{array}$ \\
\hline 50 & 8,6 & 2,6 \\
55 & 8,7 & 2,4 \\
60 & 10,4 & 3 \\
65 & 9,2 & 2,3 \\
70 & 8,6 & 2,3 \\
75 & 8,1 & 2,1 \\
80 & 7,1 & 1,7 \\
85 & 5,9 & 1,3 \\
Todas as & 8,8 & 2,5 \\
idades & &
\end{tabular}

nato genérico em Portugal - Quadro II. Os valores para o limiar de avaliação de DXA foram fixados em $2 \%$ e $0,5 \%$ acima e abaixo do limiar de intervenção das fraturas osteoporóticas major e das fraturas da anca, respetivamente.

\section{RECOMENDAÇÕES}

\section{- Recomendação 1}

A implementação de medidas gerais, não farmacológicas, de prevenção da osteoporose, como dieta, suplementação em vitamina $D$, exercício, prevenção de quedas e monitorização da utilização de terapêutica, deve ser aplicada a todas as idades, sempre que sejam identificados fatores de risco corrigíveis, independentemente da ferramenta FRAX® e DMO.

[Esta recomendação foi aprovada por todos os membros do Comité, com 17 votos em 17 e com uma média de acordo de $97 \%$ (75-100).]

\section{- Recomendação 2}

O tratamento farmacológico da osteoporose deve ser recomendado, exceto se contraindicado, a todas as pessoas com idade superior a 50 anos que já sofreram:
A. $\geq 1$ Fratura de fragilidade da anca ou $\geq 1$ fratura sintomática de fragilidade vertebral; ou

B. $\geq 2$ Fraturas de fragilidade, independentemente da localização da fratura ou de ausência de sintomas (e.g., 2 fraturas assintomáticas vertebrais).

[Esta recomendação foi aprovada por todos os membros do Comité com 17 votos em 17 e com uma média de acordo de 95,6\% (70-100).]

\section{Especificações relativas à Recomendação 2}

- Para este efeito, a fratura de fragilidade é definida como uma fratura que ocorre espontaneamente ou no seguimento de um trauma menor, i.e., queda similar ou inferior à altura de corpo, depois de serem excluídas causas patológicas de fratura, como a neoplasia.

- Esta recomendação implica que a presença de tais fraturas se sobrepõe à estimativa de risco do FRAX®Port, i.e., o tratamento deve ser considerado para estes doentes, independentemente das estimativas de risco do FRAX®Port ou da realização da DXA. Isto não implica que o FRAX® ou a DXA não possam ser realizados, na medida em que eles oferecem informação útil para uma futura investigação ou escolha de intervenções terapêuticas.

A recomendação de tratamento para as pessoas que já sofreram uma fratura de fragilidade, independentemente do FRAX®, é comum a todas as recomendações acima referidas: NOF-EUA, ${ }^{8}$ Canadá, ${ }^{11}$ França ${ }^{7}$ e Suíça. ${ }^{9}$ Este conceito é inerente às recomendações NOGG/Reino Unido. Adefinição exata varia consoante os documentos. Não se encontraram provas para propor a inclusão $\geq 2$ fraturas de fragilidade (para além das fraturas da anca ou vertebral clínica) para tratamento sem outra avaliação. Esta foi uma recomendação de consenso baseada na opinião e experiência dos autores.

\section{- Recomendação 3}

Todos os homens e mulheres portugueses com mais de 50 anos de idade devem submeter-se a uma avaliação de risco a dez anos de fratura osteoporótica, através da ferramenta FRAX®Port, com ou sem DXA.

[Esta recomendação foi aprovada por todos os membros do Comité, com 17 votos em 17 e com uma média de acordo de 95,9\% (80-100).]

\section{Especificações à Recomendação 3}

- Idealmente, a decisão de realizar a DXA deve ser baseada no FRAX®Port sem DMO, como descrito abaixo. No entanto, se já estiver disponível uma DMO recente, o seu 


\begin{tabular}{|c|c|}
\hline Doentes com as seguintes condições/doenças & $\begin{array}{c}\text { Doentes que começaram ou que se encontram a tomar os seguintes } \\
\text { medicamentos }\end{array}$ \\
\hline $\begin{array}{l}\text { Fratura de fragilidade } \leq 50^{59} \\
\text { Imobilização prolongada e paralisia }^{63-64} \\
\text { Historial de quedas }{ }^{5-6,8,11,18} \\
\text { Anorexia nervosa }{ }^{67-68} \\
\text { Deficiência de cálcio e de vitamina } D^{5,8,72-73} \\
\text { Absorção intestinal }{ }^{8,79} \\
\text { Artrite reumatoide }^{81} \\
\text { Hiperparatireoidismo }^{82-83}\end{array}$ & $\begin{array}{l}\text { Terapia de privação de androgénios } \\
\text { Glucocorticoides }^{65} \\
\text { Anticonvulsantes }^{66} \\
\text { Análogos da hormona libertadora de gonadotropinas }(\mathrm{GnRH})^{69-71} \\
\text { Inibidores de aromatase } \\
\text { Terapia antirretroviral }\end{array}$ \\
\hline
\end{tabular}

valor deve ser integrado no cálculo da FRAX®Port. O processo de decisão para o tratamento deve, neste caso, ser baseado nas recomendações 7, 8 e 9. Os valores DXA podem ser aceitáveis para este objetivo num período de até dois anos, exceto se ocorrerem entretanto acontecimentos significativos para o metabolismo dos ossos.

- Aos médicos é vivamente recomendada a adesão estrita às definições dos fatores de risco clínicos, conforme descritos no site FRAX®.

\section{- Recomendação 4}

Para estimativas do FRAX®Port, sem DXA, entre 7\% e $11 \%$ para fraturas osteoporóticas major E entre $2,0 \% \mathrm{e}$ $3,0 \%$ para fraturas da anca o índice $\mathrm{T}$ do cabeça do fémur avaliado e inserido no FRAX®Port (Figura 2).

A DXA pode ser justificada em certas condições especiais, descritas no texto.

[Esta recomendação foi aprovada com 16 votos favoráveis e 1 abstenção e uma média de acordo de 90,9\% (60-100).]

\section{Especificações à Recomendação 4}

- Para os efeitos da presente recomendação, a DMO deve ser avaliada através de absorção de raios de dupla energia (DXA).

- A coluna vertebral e o fémur proximal são os locais recomendados para a avaliação DXA. ${ }^{56}$ A DXA da coluna vertebral tem tendência para sobrestimar a DMO na presença de osteoartrite, fraturas vertebrais e outras alterações/condições calcificantes que recobrem os locais de interesse.

- O valor do índice T para a cabeça do fémur deve ser utilizado para o FRAX®Port.
- Neste contexto da decisão de tratar/não tratar, os resultados DXA devem ser considerados no quadro do FRAX®Port e não isoladamente. Este princípio implica que o diagnóstico da osteoporose ou da osteopenia baseado na densitometria não constitui, per se, uma garantia para iniciar tratamento farmacológico para a osteoporose.

- A utilização de DXA para a monitorização de terapias é controversa, raramente se justifica em intervalos de menos de 2-3 anos e pode ser dispensada de uma vez se se garantir uma adesão efetiva à terapia. (Para mais informações sobre a utilização apropriada e a interpretação da DXA, consultar referências.) $)^{18,57-58}$

- O Comité considera que a realização de DXA pode ocasionalmente ser justificada fora dos limites do FRAX, ou independentemente deles, incluindo nas condições descritas no Quadro III.

Outras condições, com uma relação menos estabelecida com a osteoporose, podem igualmente justificar a realização de DXA como parte do trabalho de diagnóstico. Estas condições incluem: mucoviscidose; Ehlers-Danlos; doença de Gaucher; doenças de depósito de glicogénio; hemocromatose; homocistinúria; hipofosfatémia; síndroma de Marfan; síndroma de Menkes; porfíria; síndroma de Riley-Day; amenorreia atlética; hiperprolactinemia; pan-hipopituitarismo; síndromas de Turner e Klinefelter; síndroma de $\mathrm{Cu}$ shing; tireotoxicose; bypass gástrico; cirurgia gastrointestinal; doença pancreática; cirrose biliar primária; hemofilia; leucemia; linfomas; gamopatias monoclonais; mioloma múltiplo; doença da anemia falciforme; mastocitose sistémica; talassemia; espondilite anquilosante; lúpus eritematoso sistémico; amiloidose; acidose metabólica crónica; 

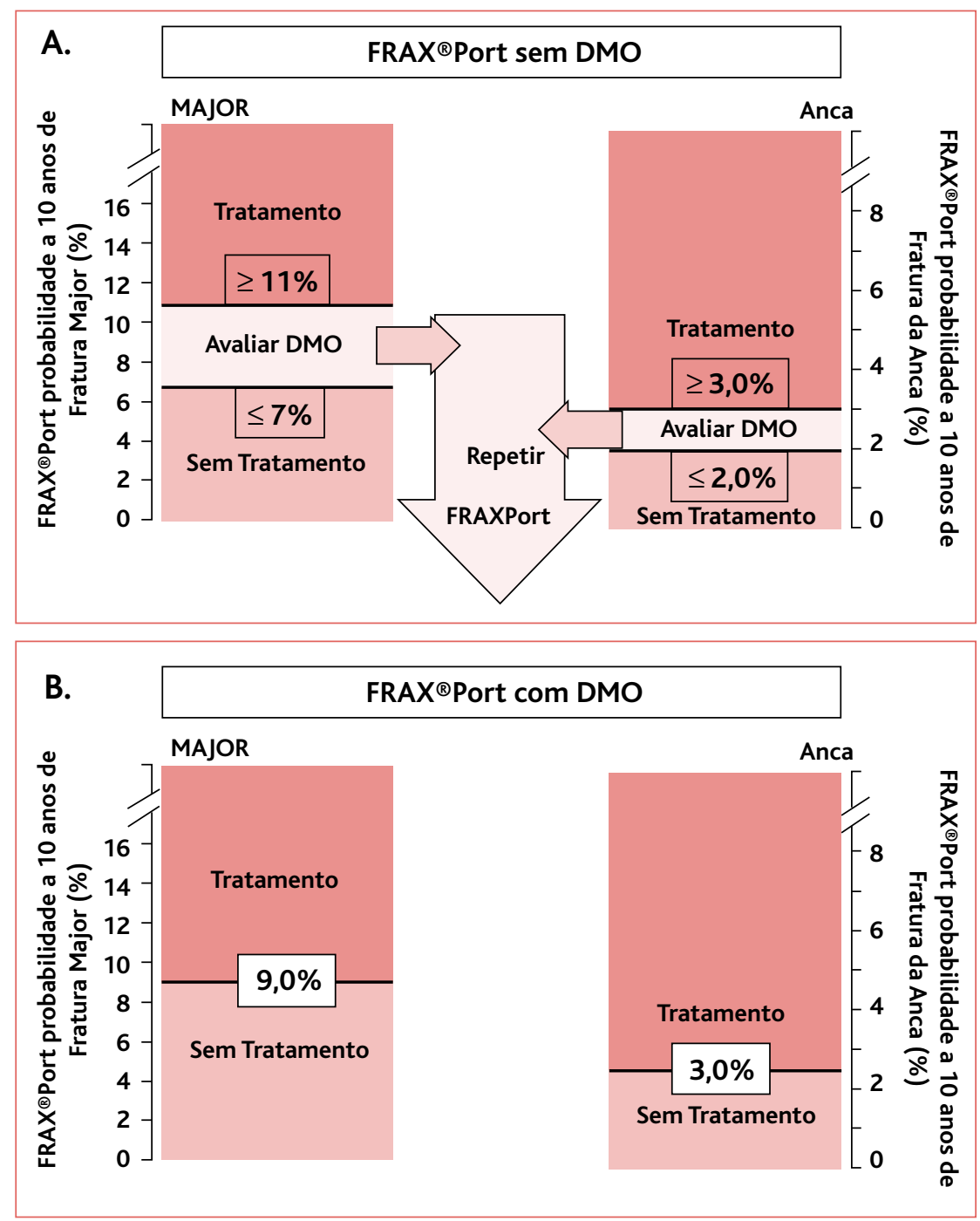

Figura 2. Utilização da FRAX ${ }^{\circledR P}$ Port para avaliar os riscos estimados a dez anos de fraturas osteoporóticas major e de fraturas da anca, para decidir do pedido de DXA e da iniciação do tratamento farmacológico para a osteoporose. A: Avaliação sem DMO. B: Avaliação com DMO.

inibidores seletivos de recaptação de serotonina; Tamoxifen $®$; tiazolidinedionas hiazolidinedionas T (como Actos®); hormonas da tiroide (em excesso).

\section{- Recomendação 5.}

5. A - Nos homens e mulheres com uma estimativa de risco de fratura (sem DMO) inferior a 7\% para as fraturas osteoporóticas major E $2 \%$ para a fratura da anca, a decisão de não tratar com agentes farmacológicos pode ser justificada, sem necessidade de realizar uma DXA. Medidas gerais de prevenção devem ser postas em prática.

[Esta recomendação foi aprovada com 16 votos favoráveis e 1 abstenção e uma média de acordo de 95\% (50100).]

5. B. Nestes casos, a avaliação com o FRAX®Port deve ser repetida com uma frequência que depende da proximidade da avaliação anterior do limite inferior de indicação para a DXA e igualmente em caso de ocorrência de mudanças significativas nos fatores de risco clínicos (Figura 2A).

[Esta recomendação foi aprovada com 16 votos favoráveis e 1 abstenção e uma média de acordo de 93,8\% (60100).]

Em relação à recomendação 5B, o Comité presume que as reavaliações FRAX®Port se justificam, em média, todos os cinco anos desde os 50 até aos

doença pulmonar obstrutiva crónica; insuficiência cardíaca congestiva; depressão; doença renal em fase terminal; hipercalciúria; escoliose idiopática; doença óssea pós-transplante; sarcoidose; diabetes mellitus tipo I.

Algumas medicações com uma relação não tão bem estabelecida com a osteoporose podem igualmente justificar a realização de uma DXA em casos especiais. Nesta categoria incluem-se: alumínio (nos antiácidos); anticoagulantes (heparina); barbitúricos; quimioterápicos contra o cancro; depomedroxiprogesterona; lítio; ciclosporina A e tracolimo; metrotrexato; nutrição parental; inibidores da bomba de protões;
70 anos e de dois em dois ou de três em três anos a partir daí, na ausência de intercorrências relevantes.

\section{- Recomendação 6.}

Nos homens e mulheres com uma estimativa de risco de fratura, sem DXA, superior a $11 \%$ para as fraturas osteoporóticas major OU de 3\% para as fraturas da anca, o tratamento farmacológico com o alendronato genérico é custo-eficaz e deve ser aconselhado (salvo em caso de contraindicações), sem necessidade de realizar DXA (Figura 2A). 
[Esta recomendação foi aprovada com 16 votos favoráveis e 1 abstenção e uma média de acordo de 95,3\% (80-100).]

\section{- Recomendação 7.}

Nos homens e mulheres com uma estimativa FRAX®Port de risco a dez anos, com DXA, igual ou superior a $9 \%$ para as fraturas osteoporóticas major OU a 2,5\% para as fraturas da anca, o tratamento farmacológico para a osteoporose com o alendronato genérico é custo-eficaz e deve ser aconselhado (salvo em caso de contraindicações) (Quadro II e Figura 2B).

[Esta recomendação foi aprovada com 17 votos em 17 e uma média de acordo de 93,2\% (60-100).]

\section{- Recomendação 8.}

A decisão de iniciar um tratamento antiosteoporótico com outros medicamentos, que não o alendronato genérico, deve ser justificada pelos respetivos limiares de intervenção de custo-eficácia (Quadro IV).

[Esta recomendação foi aprovada com 16 votos favoráveis e 1 voto contra e uma média de acordo de 88,1\% (0-100).]

\section{Especificações à recomendação 8.}

- Esta recomendação não impede a decisão de prescrever medicação com uma estimativa de risco inferior, com base numa apreciação clínica, como uma contraindicação formal em relação a alternativas menos dispendiosas ou outras condições que fazem com que a escolha selecionada seja particularmente apropriada. O médico pode também decidir adotar uma diferente disponibilidade de tratar.

[Esta especificação foi aprovada com 16 votos favoráveis e 1 abstenção e uma média de acordo de 99,3\% (90-100).]

O custo por QALY associado a diferentes medicações é afetado pelo seu custo e eficiência nos diferentes locais clínicos. O Quadro IV apresenta os níveis de avaliação de risco a partir dos quais o tratamento com ácido zoledrónico, denosumab e teriparatida são custo-eficazes em comparação com nenhum tratamento e podem, portanto, ser recomendados com base na relação de custo-eficácia, como descrito por Marques e colaboradores. ${ }^{55}$

Os autores sublinham que não estão disponíveis dados nacionais sobre os limiares de custo-eficácia para outros medicamentos. A única alternativa é a da extrapolação com base nos indicadores de eficácia, persistência e custo para essas outras drogas alternativas quando comparadas com as opções estudadas.

\section{- Recomendação 9.}

9. A. Nos homens e mulheres com uma estimativa FRAX®Port de risco a dez anos, com DXA, inferior a 9\% para as fraturas osteoporóticas major $\mathrm{E}$ inferior a 2,5\% para as fraturas da anca, os agentes farmacológicos não são custo-eficazes e a decisão de não os utilizar pode ser justificada. As medidas gerais de prevenção aplicáveis devem ser postas em prática.

[Esta recomendação foi aprovada por todos os membros do Comité e uma média de acordo de 96,5\% (80-100).]

9 B. Nestes doentes, as avaliações DXA e FRAX®Port devem ser repetidas todos os dois anos ou sempre que os fatores de risco sofram uma alteração significativa (Figura 2). A DXA pode não ser necessária se os valores de uma DMO anteriores forem tranquilizadores.

[Esta recomendação foi aprovada com 16 votos favoráveis e 1 abstenção e uma média de acordo de 92,8\% (75100).]

\section{- Recomendação 10.}

Quando recorrerem ao FRAX®Port para a implementação destas recomendações, os profissionais de saúde devem estar conscientes das limitações deste instrumento e ponderarem a necessidade de ajustar as estimativas de risco fornecidas por esta ferramenta em situações específicas que se descrevem no texto. 
[Esta recomendação foi aprovada com 17 votos em 17 e uma média de acordo de $97,6 \%(70-100)$.]

\section{Especificações à recomendação 10.}

1. As limitações do FRAX®Port são as mesmas do FRAX®. Algumas destas limitações poderão vir a ser resolvidas em futuras revisões desta ferramenta.

2. O FRAX® não tem em consideração o número de fraturas de fragilidade anteriores. ${ }^{18}$ Contudo, esta limitação é resolvida pela decisão do Comité de recomendar a fratura de fragilidade prévia como um critério independente para começar o tratamento.

3. O FRAX® não foi validado para ser utilizado em doentes sob tratamento osteoporótico ou para monitorização dos efeitos do tratamento. ${ }^{18}$

[Esta especificação foi aprovada por 16 votos favoráveis e 1 abstenção e uma média de acordo de $100 \%$.]

4. As quedas são um importante fator clínico de risco para as fraturas e não estão incluídas na ferramenta FRAX ${ }^{\circledR} .{ }^{9}$ Não é possível apresentar uma recomendação formal para este efeito, em virtude da falta de provas científicas apropriadas. Os melhores valores de referência que podem ser fornecidos baseiam-se em cálculos realizados com o QFracture ${ }^{\circledR} 2013,{ }^{82-83}$ um instrumento validado e preciso de estimação do risco de fratura, que toma em consideração as quedas. Neste contexto, a presença de um «historial de quedas» multiplica por um fator de cerca de 1,5 as estimativas de risco de fratura a dez anos, calculadas sem esta ferramenta.

[Esta especificação foi aprovada por 17 votos favoráveis e uma média de acordo de 92,1\% (0-100).]

1. A ferramenta FRAX não tem em consideração a dose de corticosteroides acima de $5 \mathrm{mg}$ de um equivalente da Prednisolona para mais de três meses. O Comité recomenda que as probabilidades a dez anos de fratura da anca ou de fratura osteoporótica sejam ajustadas de acordo com a dose de glucocorticoides descrita no Quadro V. Por falta de evidência apropriada, não é possível propor ajustamentos em relação à duração do tratamento. [Esta especificação foi aprovada com 16 votos favoráveis e 1 abstenção e uma média de acordo de 87,5\% (50-100).]

\section{O algoritmo $\mathrm{FRAX}^{\circledR}$ utiliza o índice T para a DMO do colo} do fémur e não tem em conta a DMO da coluna lom- bar. No entanto, quando há uma discordância muito grande ( > 1DP entre o índice T do colo do fémur e a coluna lombar, propõe-se que:

O médico possa aumentar/diminuir em $10 \%$ a estimativa FRAX ${ }^{\circledR}$ para as fraturas osteoporóticas major para cada diferença arredondada do índice T entre a coluna lombar e o colo do fémur. ${ }^{5,85}$

Por exemplo, se o índice $\mathrm{T}$ do colo do fémur é igual a -1,5 e o índice $\mathrm{T}$ da coluna lombar é igual a -2,8, a estimativa FRAX ${ }^{\circledR}$ para as fraturas osteoporóticas major deve ser aumentada de $10 \%$ (e.g., de $7 \%$ para $7,7 \%$ ). Se os valores forem -1,5 e -1,9, respetivamente, nenhumas alterações devem ser introduzidas (diferença $<0,5 \mathrm{~T}$ ). Se o índice $\mathrm{T}$ do colo do fémur é igual a -2,3 e o da coluna lombar $-3,9$, a diferença $(1,6)$ é arredondada para o índice 2 $\mathrm{T}$ e as estimativas de risco de fratura osteoporótica major devem ser aumentadas de $20 \%$ (e.g., de $8 \%$ para $9,6 \%$, justificando medicação segundo as presentes recomendações).

Tal como em todas as outras circunstâncias, é importante garantir a qualidade e a validade da DXA da coluna lombar.

[Esta especificação foi aprovada com 17 votos favoráveis e uma média de acordo de 91,5\% (75-100).]

Na Figura 3 apresenta-se um diagrama integrado e simplificado sobre as decisões de tratamento e a avaliação DXA, de acordo com as presentes recomendações. Tenha em mente que os limiares de intervenção se baseiam em cálculos para o alendronato genérico. Para os adaptar a outras terapêuticas, refira-se a recomendação 8.

Seguimento - Repetir as avaliações, como sugerido nas recomendações 5B e 9B. 


\section{DISCUSSÃO E OBSERVAÇÕES FINAIS}

Foram desenvolvidas dez recomendações para os doentes portugueses sobre quem deve receber tratamento para a osteoporose e quem deve ser examinado com DXA na prática clínica diária, baseadas nos princípios de orientação consensuais e nas avaliações epidemiológicas e económicas no contexto português (Quadro I). Estas recomendações são práticas, baseiam-se em provas e foram apoiadas por um painel de especialistas e de representantes das instituições científicas e das associações de doentes portuguesas ligadas à osteoporose.

Tanto quanto possível, as recomendações basearam-se em evidência, tendo estas sido suplementadas por decisões colegiais dos especialistas em caso de inexistência de provas decisivas. Foram desenvolvidos esforços consideráveis para manter as recomendações tão simples quanto possível, mas também compreensíveis, isto é, suscetíveis de responder à maior parte das necessidades da prática clínica.

Estas recomendações fornecem uma base muito mais sólida e fundamentada para as decisões de tratamento do que a consideração exclusiva da Densidade Mineral Óssea (DMO) ou a ponderação subjetiva por parte dos médicos dos fatores de risco clínico. A ferramenta FRAX® permite a integração de um grande número de fatores de risco clínico para fraturas, de relevância comprovada e cujo impacto foi estimado por meta-análise. Além disso, a versão portuguesa do FRAX incorpora a epidemiologia atual das fraturas de fragilidade e mortalidade na população-alvo. No nosso contexto epidemiológico e económico atual, tomar em consideração as análises da relação custo-eficácia das intervenções responde à responsabilidade de utilizar judiciosamente os recursos limitados disponíveis para a saúde. Estes cálculos foram efetuados com recurso a mo- delos económicos de última geração e a aconselhamento económico de reconhecido prestígio. A disponibilidade de pagar que foi adotada segue as recomendações internacionais.

Um certo grau de arbitrariedade foi utilizado no estabelecimento de limiares de intervenção similares para todas as idades, apesar de haver uma variabilidade considerável entre os grupos etários. $\mathrm{O}$ mesmo se aplica ao valor adotado para a Disponibilidade de Pagar: alguns médicos podem ter um ponto de vista diferente e a Disponibilidade de Pagar pode sofrer alterações de acordo com o PNB e as políticas nacionais de saúde. Os utilizadores experientes podem querer desenvolver uma definição mais precisa dos limiares de custo-eficácia para casos individuais específicos, tendo em conta a idade do doente, a medicação considerada e uma Disponibilidade de Pagar 
da sua livre escolha. Pode ser feito através da utilização de uma ferramenta específica que foi disponibilizada por Marques e colaboradores: ${ }^{55} \mathrm{https} / /$ dl.dropboxusercontent.com/u/4287154/OsteoporoseThrCalc/ThreshComputationPortugalFINAL.xlsm

Estas recomendações representam uma importante mudança de paradigma, que se tornou possível com o desenvolvimento do FRAX® e da sua adaptação portuguesa e com as avaliações económicas a que se faz referência acima. Acredita-se que é imenso o potencial desta mudança que visa uma utilização mais eficiente dos recursos humanos e financeiros no combate ao problema crescente que a epidemia de fraturas osteoporóticas representa. No entanto, tudo depende da utilização que os profissionais de saúde, tanto individual como coletivamente, derem a estas novas ferramentas. Espera-se que a adoção destas recomendações por todos os especialistas e instituições representadas contribua para aumentar a sua divulgação e implementação na prática clínica nacional, reforçando, assim, o seu potencial de promoção do progresso relativamente aos padrões atuais de gestão da osteoporose no nosso país.

Manifesta-se desde já o reconhecimento a todos os profissionais de saúde que estejam dispostos a partilhar as suas opiniões e experiências sobre a utilização destas recomendações e que sugiram formas de melhorar o seu desempenho para benefício da saúde pública (reuma@huc. min-saude.pt).

\section{REFERÊNCIAS BIBLIOGRÁFICAS}

1. de Pina MF, Alves SM, Barbosa M, Barros H. Hip fractures cluster in space: an epidemiological analysis in Portugal. Osteoporos Int. 2008;19(12):1797-804.

2. Marques AA, Lourenço $O$, Pereira da Silva JA. The burden of osteoporotic hip fractures in Portugal: costs, quality of life and mortality. Osteoporos Int. In press.

3. Instituto Nacional de Estatística. Conta satélite da saúde 2000-2012. Lisboa: INE; 2012.

4. Rodrigues VM. Epidemiologia de fraturas osteoporóticas no serviço de urgência dos CHUC [Internet]. Coimbra: Faculdade de Medicina da Universidade de Coimbra; 2015. Available from: https://estudogeral.sib.uc.pt/bitstream/10316/ 30459/1/tese\%20final\%20revis\%C3\%A3o.pdf

5. Kanis JA, McCloskey EV, Johansson H, Cooper C, Rizzoli R, Reginster JY. European guidance for the diagnosis and management of osteoporosis in postmenopausal women. Osteoporos Int. 2013;24(1):23-57.

6. Tavares V, Canhão H, Gomes JA, Simões E, Romeu JC, Coelho P, et al. Recomendações para o diagnóstico e terapêutica da osteoporose [Recommendations for the diagnosis and management of osteoporosis]. Acta Reumatol Port. 2007;32(1):49-59. Portuguese

7. Briot K, Cortet B, Thomas T, Audran M, Blain H, Breuil V, et al. 2012 Update of French guidelines for the pharmacological treatment of postmenopausal osteoporosis. Joint, Bone Spine. 2012;79(3):304-13.

8. Cosman F, de Beur SJ, LeBoff MS, Lewiecki EM, Tanner B, Randall S, et al. Clinician's guide to prevention and treatment of osteoporosis. Osteoporos Int. 2014;25(10):2359-81.
9. Lippuner K, Johansson H, Borgstrom F, Kanis JA, Rizzoli R. Cost-effective intervention thresholds against osteoporotic fractures based on FRAX ${ }^{\circledR}$ in Switzerland. Osteoporos Int. 2012;23(11):2579-89.

10. Makras P, Athanasakis K, Boubouchairopoulou N, Rizou S, Anastasilakis AD, Kyriopoulos J, et al. Cost-effective osteoporosis treatment thresholds in Greece. Osteoporos Int. 2015;26(7):1949-57.

11. PapaioannouA, Morin S, Cheung AM,Atkinson S, Brown JP, Feldman S, et al. 2010 Clinical practice guidelines for the diagnosis and management of osteoporosis in Canada: summary. CMAJ. 2010;182(17):1864-73.

12. Tosteson AN, Melton LJ 3rd, Dawson-Hughes B, Baim S, Favus MJ, Khosla S, et al. Cost-effective osteoporosis treatment thresholds: the United States perspective. Osteoporos Int. 2008;19(4):437-47.

13. Schuit SC, van der Klift M, Weel AE, de Laet CE, Burger H, Seeman E, et al. Fracture incidence and association with bone mineral density in elderly men and women: the Rotterdam study. Bone. 2004;34(1):195-202.

14. Kanis JA, Oden A, Johnell O, Johansson H, De Laet C, Brown J, et al. The use of clinical risk factors enhances the performance of BMD in the prediction of hip and osteoporotic fractures in men and women. Osteoporos Int. 2007;18(8):1033-46.

15. Marques A, Mota A, Canhão H, Romeu JC, Machado P, Ruano A, et al. A FRAX model for the estimation of osteoporotic fracture probability in Portugal. Acta Reumatol Port. 2013;38(2):104-12.

16. Kanis JA. Diagnosis of osteoporosis and assessment of fracture risk. Lancet. 2002;359(9321):1929-36.

17. Johansson H, Kanis JA, Oden A, Johnell O, McCloskey E. BMD, clinical risk factors and their combination for hip fracture prevention. Osteoporos Int. 2009;20(10):1675-82.

18. Kanis JA, Hans D, Cooper C, Baim S, Bilezikian JP, Binkley N, et al. Interpretation and use of FRAX in clinical practice. Osteoporosis Int. 2011;22(9):2395-411.

19. Marques A, Ferreira RJ, Santos E, Loza E, Carmona L, da Silva JA. The accuracy of osteoporotic fracture risk prediction tools: a systematic review and meta-analysis. Ann Rheum Dis. 2015;74(11):1958-67.

20. Kanis JA, Oden A, Johnell O, Johansson H, De Laet C, Brown J, et al. The use of clinical risk factors enhances the performance of BMD in the prediction of hip and osteoporotic fractures in men and women. Osteoporos Int. 2007;18(8):1033-46.

21. Azagra R, Roca G, Martín-Sánchez JC, Casado E, Encabo G, Zwart M, et al. Umbrales de FRAX ${ }^{\circledR}$ para identificar personas con alto o bajo riesgo de fractura osteoporótica en población femenina española [FRAX® thresholds to identify people with high or low risk of osteoporotic fracture in Spanish female population]. Med Clin (Barc). 2015;144(1):1-8. Spanish

22. Bolland MJ, Siu AT, Mason BH, Horne AM, Ames RW, Grey AB, et al. Evaluation of the FRAX and Garvan fracture risk calculators in older women. J Bone Miner Res. 2011;26(2):420-7.

23. Brennan SL, Leslie WD, Lix LM, Johansson H, Oden A, McCloskey E, et al. FRAX provides robust fracture prediction regardless of socioeconomic status. Osteoporos Int. 2014;25(1):61-9.

24. Cheung EY, Bow CH, Cheung CL, Soong C, Yeung S, Loong C, et al. Discriminative value of FRAX for fracture prediction in a cohort of Chinese postmenopausal women. Osteoporos Int. 2012;23(3):871-8.

25. Cummins NM, Poku EK, Towler MR, O'Driscoll OM, Ralston SH. Clinical risk factors for osteoporosis in Ireland and the UK: a comparison of FRAX and QFractureScores. Calcif Tissue Int. 2011;89(2):172-7.

26. Donaldson MG, Palermo L, Schousboe JT, Ensrud KE, Hochberg MC, Cummings SR. FRAX and risk of vertebral fractures: the fracture intervention trial. J Bone Miner Res. 2009;24(11):1793-9.

27. Ensrud KE, Lui LY, Taylor BC, Schousboe JT, Donaldson MG, Fink HA, et al.A comparison of prediction models for fractures in older women: is more better? Arch Intern Med. 2009;169(22):2087-94.

28. Ettinger B, Ensrud KE, Blackwell T, Curtis JR, Lapidus JA, Orwoll ES. Performance 
of FRAX in a cohort of community-dwelling, ambulatory older men: the Osteoporotic Fractures in Men (MrOS) study. Osteoporos Int. 2013;24(4):1185-93.

29. Fraser LA, Langsetmo L, Berger C, loannidis G, Goltzman D, Adachi JD, et al. Fracture prediction and calibration of a Canadian FRAX ${ }^{\circledR}$ tool: a population-based report from CaMos. Osteoporos Int. 2011;22(3):829-37.

30. Friis-Holmberg T, Rubin KH, Brixen K, Tolstrup JS, Bech M. Fracture risk prediction using phalangeal bone mineral density or FRAX ${ }^{\circledR}$ ? A Danish cohort study on men and women. J Clin Densitom. 2014;17(1):7-15.

31. González-Macias J, Marin F, Vila J, Díez-Pérez A. Probability of fractures predicted by FRAX ${ }^{\circledR}$ and observed incidence in the Spanish ECOSAP Study cohort. Bone. 2012;50(1):373-7.

32. Hillier TA, Cauley JA, Rizzo JH, Pedula KL, Ensrud KE, Bauer DC, et al. WHO absolute fracture risk models (FRAX): do clinical risk factors improve fracture prediction in older women without osteoporosis? J Bone Miner Res. 2011;26(8): 1774-82.

33. Langsetmo L, Nguyen TV, Nguyen ND, Kovacs CS, Prior JC, Center JR, et al. Independent external validation of nomograms for predicting risk of low-trauma fracture and hip fracture. CMAJ. 2011;183(2):E107-14.

34. LeslieWD, Lix LM, Johansson H, Oden A, McCloskey E, Kanis JA. Independent clinical validation of a Canadian FRAX tool: fracture prediction and model calibration. J Bone Miner Res. 2010;25(11):2350-8.

35. Premaor M, Parker RA, Cummings S, Ensrud K, Cauley JA, Lui LY, et al. Predictive value of FRAX for fracture in obese older women. J Bone Miner Res. 2013;28(1):188-95.

36. Pressman AR, Lo JC, Chandra M, Ettinger B. Methods for assessing fracture risk prediction models: experience with FRAX in a large integrated health care delivery system. J Clin Densitom. 2011;14(4):407-15.

37. Sambrook PN, Flahive J, Hooven FH, Boonen S, Chapurlat R, Lindsay R, et al. Predicting fractures in an international cohort using risk factor algorithms without BMD. J Bone Miner Res. 2011;26(11):2770-7.

38. Sandhu SK, Nguyen ND, Center JR, Pocock NA, Eisman JA, Nguyen TV. Prognosis of fracture: evaluation of predictive accuracy of the FRAX algorithm and Garvan nomogram. Osteoporos Int. 2010;21(5):863-71.

39. Sornay-Rendu E, Munoz F, Delmas PD, Chapurlat RD. The FRAX tool in French women: how well does it describe the real incidence of fracture in the OFELY cohort. J Bone Miner Res. 2010;25(10):2101-7.

40. Sund R, Honkanen R, Johansson H, Odén A, McCloskey E, Kanis J, et al. Evaluation of the FRAX model for hip fracture predictions in the population-based Kuopio Osteoporosis Risk Factor and Prevention Study (OSTPRE). Calcif Tissue Int. 2014;95(1):39-45.

41. Tamaki J, Iki M, Kadowaki E, Sato Y, Kajita E, Kagamimori S, et al. Fracture risk prediction using FRAX ${ }^{\circledR}$ : a 10-year follow-up survey of the Japanese Population-Based Osteoporosis (JPOS) cohort study. Osteoporosis Int. 2011;22(12):3037-45

42. Tebe Cordomí C, del Río LM, Di Gregorio S, Casas L, Estrada MD, Kotzeva A, et al. Validation of the FRAX predictive model for major osteoporotic fracture in a historical cohort of spanish women. J Clin Densitom. 2013;16(2):231-7.

43. Trémollieres FA, Pouillès JM, Drewniak N, Laparra J, Ribot CA, Dargent-Molina $P$. Fracture risk prediction using BMD and clinical risk factors in early postmenopausal women: sensitivity of the WHO FRAX tool. J Bone Miner Res. 2010;25(5):1002-9.

44. Marques AF, Ferreira RJ, Santos E, Losa E, Carmona L, da Silva JA, et al. The accuracy of osteoporotic fracture risk prediction tools: a systematic review and meta-analysis. Ann Rheum Dis. 2015;74(11):1958-67.

45. Kanis JA, McCloskey EV, Johansson H, Strom O, Borgstrom F, Oden A. Case finding for the management of osteoporosis with FRAX: assessment and intervention thresholds for the UK. Osteoporos Int. 2008;19(10):1395-408.

46. Johnell O, Oden A, Caulin F, Kanis JA. Acute and long-term increase in fracture risk after hospitalization for vertebral fracture. Osteoporos Int. 2001;12(3):207-14.

47. Klotzbuecher CM, Ross PD, Landsman PB, AbbottTA 3rd, Berger M. Patients with prior fractures have an increased risk of future fractures: a summary of the literature and statistical synthesis. J Bone Miner Res. 2000;15(4):721-39.

48. Cuddihy MT, Gabriel SE, Crowson CS, O'Fallon WM, Melton LJ 3rd. Forearm fractures as predictors of subsequent osteoporotic fractures. Osteoporos Int. 1999;9(6):469-75.

49. van Geel TA, van Helden S, Geusens PP, Winkens B, Dinant GJ. Clinical subsequent fractures cluster in time after first fractures. Ann Rheum Dis. 2009;68(1):99-102.

50. Giangregorio LM, Leslie WD. Time since prior fracture is a risk modifier for 10year osteoporotic fractures. J Bone Miner Res. 2010;25(6):1400-5.

51. Borgström F, Johnell O, Kanis JA, Jönsson B, Rehnberg C. At what hip fracture risk is it cost-effective to treat? International intervention thresholds for the treatment of osteoporosis. Osteoporos Int. 2006;17(10):1459-71.

52. Marseille E, Larson B, Kazi DS, Kahn JG, Rosen S. Thresholds for the cost-effectiveness of interventions: alternative approaches. Bull World Health Organ. 2015;93(2):118-124.

53. European Commission, Economic and Financial Affairs. AMECO: variables. European Commission; 2015 [cited 2015 Feb 7]. Available from: http://ec.europa.eu/economy_finance/ameco/user/serie/ResultSerie.cfm

54. Borgström F, Ström O, Kleman M, McCloskey E, Johansson H, Odén A, et al. Costeffectiveness of bazedoxifene incorporating the FRAX ${ }^{\circledR}$ algorithm in a European perspective. Osteoporos Int. 2011;22(3):955-65.

55. Marques A, Lourenco O, Ortsater G, et al. Cost-Effectiveness of Intervention Thresholds for the Treatment of Osteoporosis Based on FRAX ${ }^{\circledR}$ in Portugal. Calcif Tissue Int 2016;99(2):131-41.

56. Blake GM, Fogelman I.The role of DXA bone density scans in the diagnosis and treatment of osteoporosis. Postgrad Med J. 2007;83(982):509-17.

57. Schousboe JT, Shepherd JA, Bilezikian JP, Baim S. Executive summary of the 2013 International Society for Clinical Densitometry Position Development Conference on bone densitometry. J Clin Densitom. 2013;16(4):455-66.

58. Watts NB. Fundamentals and pitfalls of bone densitometry using dual-energy X-ray absorptiometry (DXA). Osteoporos Int. 2004;15(11):847-54.

59. Hernlund E, Svedbom A, Ivergård M, Compston J, Cooper C, Stenmark J, et al. Osteoporosis in the European Union: medical management, epidemiology and economic burden. A report prepared in collaboration with the International Osteoporosis Foundation (IOF) and the European Federation of Pharmaceutical Industry Associations (EFPIA). Arch Osteoporos. 2013;8:136.

60. Ding H, Yang L, Du W, Teng Y, Fu SJ, Tao Y, et al. Bisphosphonates for osteoporosis in nonmetastatic prostate cancer patients receiving androgen-deprivation therapy: a systematic review and meta-analysis. Asian Pac J Cancer Prev. 2013;14(5):3337-43.

61. Serpa Neto A, Tobias-Machado M, Esteves MA, Senra MD, Wroclawski ML, Fonseca FL, et al. A systematic review and meta-analysis of bone metabolism in prostate adenocarcinoma. BMC Urol. 2010;10:9.

62. Lattouf JB, Saad F. Bone complications of androgen deprivation therapy: screening, prevention, and treatment. Curr Opin Urol. 2010;20(3):247-52.

63. Charmetant C, Phaner V, Condemine A, Calmels P. Diagnosis and treatment of osteoporosis in spinal cord injury patients: a literature review. Ann Phys Rehabil Med. 2010;53(10):655-68.

64. Smith E, Carroll A. Bone mineral density in adults disabled through acquired neurological conditions: a review. J Clin Densitom. 2011;14(2):85-94.

65. Kanis JA, Johansson H, Oden A, Johnell O, de Laet C, Melton III LJ, et al. A metaanalysis of prior corticosteroid use and fracture risk. J Bone Miner Res. 2004;19(6):893-9.

66. Lee RH, Lyles KW, Cólon-Emeric C.A review of the effect of anticonvulsant medications on bone mineral density and fracture risk. Am J Geriatr Pharmacother. 2010;8(1):34-46.

67. Kueper J, Beyth S, Liebergall M, Kaplan L, Schroeder JE. Evidence for the adverse effect of starvation on bone quality: a review of the literature. Int J Endocrinol. 2015;2015:ID628740. 
68. Fazeli PK, Klibanski A.Anorexia nervosa and bone metabolism. Bone. 2014;66:3945.

69. Sagsveen M, Farmer JE, Prentice A, Breeze A. Gonadotrophin-releasing hormone analogues for endometriosis: bone mineral density. Cochrane Database Syst Rev. 2003(4):CD001297.

70. Wu D, Hu M, Hong L, Hong S, Ding W, Min J, et al. Clinical efficacy of add-back therapy in treatment of endometriosis: a meta-analysis. Arch Gynecol Obstetr. 2014;290(3):513-23

71. Fernandez H, Lucas C, Hédon B, Meyer JL, Mayenga JM, Roux C. One year comparison between two add-back therapies in patients treated with a GnRH agonist for symptomatic endometriosis: a randomized double-blind trial. Hum Reprod. 2004;19(6):1465-71.

72. Reid IR, Bolland MJ, Grey A. Effects of vitamin D supplements on bone mineral density: a systematic review and meta-analysis. Lancet. 2014;383(9912):14655.

73. Boonen S, Bischoff-Ferrari HA, Cooper C, Lips P, Ljunggren O, Meunier PJ, et al. Addressing the musculoskeletal components of fracture risk with calcium and vitamin D: a review of the evidence. Calcif Tissue Int. 2006;78(5):257-70.

74. Bauer M, Bryce J, Hadji P.Aromatase inhibitor-associated bone loss and its management with bisphosphonates in patients with breast cancer. Breast Cancer. 2012;4:91-101.

75. Hadji P, Aapro MS, Body JJ, Bundred NJ, Brufsky A, Coleman RE, et al. Management of aromatase inhibitor-associated bone loss in postmenopausal women with breast cancer: practical guidance for prevention and treatment. Ann Oncol. 2011;22(12):2546-55.

76. Ligibel JA, O'Malley AJ, Fisher M, Daniel GW, Winer EP, Keating NL. Patterns of bone density evaluation in a community population treated with aromatase inhibitors. Breast Cancer Res Treat. 2012;134(3):1305-13.

77. Pavone ME, Bulun SE. Aromatase inhibitors for the treatment of endometriosis. Fertil Steril. 2012;98(6):1370-9.

78. Usluogullari B, Duvan CZ, Usluogullari CA. Use of aromatase inhibitors in practice of gynecology. J Ovarian Res. 2015;8:4.

79. Vestergaard P. Bone loss associated with gastrointestinal disease: prevalence and pathogenesis. Eur J Gastroenterol Hepatol. 2003;15(8):851-6.
80. Brown TT, Ruppe MD, Kassner R, Kumar P, Kehoe T, Dobs AS, et al. Reduced bone mineral density in human immunodeficiency virus-infected patients and its association with increased central adiposity and postload hyperglycemia. J Clin Endocrinol Metab. 2004;89(3):1200-6.

81. Kanis JA, Borgstrom F, De Laet $C$, Johansson $H$, Johnell $O$, Jonsson $B$, et al. Assessment of fracture risk. Osteoporos Int. 2005;16(6):581-9.

82. Khan A, GreyA, Shoback D. Medical management of asymptomatic primary hyperparathyroidism: proceedings of the third international workshop. J Clin Endocrinol Metab. 2009;94(2):373-81.

83. Marcocci C, Bollerslev J, Khan AA, Shoback DM. Medical management of primary hyperparathyroidism: proceedings of the fourth International Workshop on the Management of Asymptomatic Primary Hyperparathyroidism. J Clin Endocrinol Metab. 2014;99(10):3607-18.

84. Kanis JA, Johansson H, OdenA, McCloskey EV. Guidance for the adjustment of FRAX according to the dose of glucocorticoids. Osteoporos Int. 2011;22(3):809-16.

85. Leslie WD, Lix LM, Johansson H, Oden A, McCloskey E, Kanis JA. Spine-hip discordance and fracture risk assessment: a physician-friendly FRAX enhancement. Osteoporos Int. 2011;22(3):839-47.

\section{CONFLITO DE INTERESSES}

Os autores declaram não ter conflitos de interesses.

\section{ENDEREÇO PARA CORRESPONDÊNCIA}

José António Pereira da Silva

Serviço de Reumatologia, $7^{\circ}$ piso,

Centro Hospitalar e Universitário de Coimbra

Coimbra, Portugal

E-mail: amarques@reumahuc.org

Este artigo foi co-publicado no n. ${ }^{\circ} 4$ de 2016 da Acta Reumatológica Portuguesa (Acta Reumatol Port; 41(4):305-318 Out-Dez 2016);

This article has been copublished in the issue no. 4 of Acta Reumatológica Portuguesa (Acta Reumatol Port; 41(4):305-318 Oct-Dec 2016)

\section{ABSTRACT}

\section{PORTUGUESE RECOMMENDATIONS FOR ORDERING BONE DENSITOMETRY MEASUREMENT AND INDICATIONS FOR TREATMENT TO PREVENT OSTEOPOROTIC FRACTURES}

Objective: To establish Portuguese recommendations regarding the indications for ordering bone densitometry measurement to (DXA) and for starting treatment to prevent osteoporotic fractures.

Methods: A multidisciplinary panel, representing a wide range of medical specialties and patient associations related to osteoporosis, as well as national experts in this field and in health economics, met to develop recommendations based on available evidence and expert consensus. Recently obtained data on the epidemiologic, economic and quality-of-life aspects of osteoporotic fractures in Portugal were used to support decisions.

Results: Ten recommendations were developed covering the issues of indications for ordering DXA measurements and whom to treat with anti-fracture medications. Thresholds for assessment and intervention are based on the cost-effectiveness analysis of interventions at different thresholds of a ten-year probability of osteoporotic fractures, calculated with the Portuguese version of FRAX ${ }^{\circledR}$ (FRAX ${ }^{\circledR}$ Port), and taking into account Portuguese epidemiologic and economic data. Limitations of FRAX ${ }^{\circledR}$ are highlighted and guidance for appropriate adjustment is provided, when possible.

Conclusions: Cost-effectiveness thresholds for DXA examination and drug intervention aiming at fragility fracture prevention are provided for the Portuguese population. These are practical, based on national epidemiological and economic data, evidence-based, and supported by a multidisciplinary panel of experts and scientific societies. Implementation of these recommendations has the possibility to assure the most effective use of health resources in the prevention of osteoporotic fractures in Portugal. 